\title{
Utility of Outpatient Syndromic Data for Monitoring Influenza-like IIIness
}

\author{
Jill K. Baber* and Michelle Feist \\ Division of Disease Control, North Dakota Department of Health, Bismarck, ND, USA
}

\section{Objective}

To explore how outpatient and urgent care syndromic surveillance for influenza-like illness (ILI) compare with emergency department syndromic ILI and other seasonal ILI surveillance indicators.

\section{Introduction}

The North Dakota Department of Health (NDDoH) collects outpatient ILI data through North Dakota Influenza-like Illness Network (ND ILINet), providing situational awareness regarding the percent of visits for ILI at sentinel sites across the state. Because of increased clinic staff time devoted to electronic health initiatives and an expanding population, we have found sentinel sites have been harder to maintain in recent years, and the number of participating sentinel sites has decreased. Outpatient sentinel surveillance for influenza is an important component of influenza surveillance because hospital and death surveillance does not capture the full spectrum of influenza illness.

Syndromic surveillance (SyS) is another possible source of information for outpatient ILI that can be used for situational awareness during the influenza season; one benefit of SyS is that it can provide more timely information than traditional outpatient ILI surveillance [1,2]. The NDDoH collects SyS data from hospitals (emergency department and inpatient visits) and outpatient clinics, including urgent and primary care locations. Visits include chief complaint and/or diagnosis code data. This data is sent to the BioSense 2.0 SyS platform. We compared our outpatient SyS ILI with our ND ILINet and reported influenza cases, and included hospital and combined SyS ILI for comparison.

\section{Methods}

Weekly rates from ND ILINet, SyS ILI, and counts of reported cases from the influenza season (annual weeks 40 through 20) for the 2014-2015 and 2015-2016 seasons were compiled. Syndromic categories for outpatient, hospital (emergency department and inpatient), and combined hospital and outpatient data were created, and the BioSense 2.0 definition for ILI was used. These included data from 127,050 outpatient and 323,318 hospital visits for 2014-15, and 124,597 outpatient and 424,097 hospital visits for 2015-16. Because influenza is a reportable condition in North Dakota, case data is routinely used to represent the seasonal influenza trend, and is useful when other respiratory viruses are circulating. A Pearson Correlation Coefficient was calculated on all variables using SAS 9.4. Alpha was set to 0.05 . There was no overlap between the outpatient clinics providing syndromic surveillance data and clinics participating in ND ILINet.

\section{Results}

All outpatient, hospital, and combined outpatient and hospital ILI rates from SyS data were positively and significantly correlated with both ND ILINet rates and influenza case counts (Table 1). The correlation between outpatient SyS ILI rates and traditional influenza indicators was lower than for hospital SyS ILI rates for both years, with correlation coefficients ranging from $0.38-0.48$ and $0.56-0.92$, respectively. Generally SyS data was more highly correlated with case counts than ND ILINET rates. For the 2014-15 season, hospital
SyS data was the most strongly correlated with traditional influenza indicators. For 2015-16, combined SyS data was the most strongly correlated. Visual inspection of the chief complaint data for ILI visits found a significant number of gastrointestinal visits that included the phrase "flu-like illness" in both outpatient and hospital SyS data.

\section{Conclusions}

Although correlation coefficients were lower for outpatient SyS ILI rates, they are significant enough to be included in our ongoing influenza surveillance. One possible confounding factor for the relationship between ED surveillance and reported cases is that people with more severe illness may be more likely to be tested for influenza, and may be more likely to seek medical attention at a hospital setting. This may explain why hospital SyS data provided the strongest correlation during the 2014-15 season, a season with higher rates of more severe illness than 2015-16. The combination of outpatient data and hospital data provided the strongest correlation for the 2015-16 influenza season, indicating the addition of outpatient data, which may increase representativeness of ILI data, may be beneficial to SyS ILI surveillance. We used an existing ILI syndrome from the BioSense 2.0 tool, and revising this syndrome may improve correlations between SyS ILI and ND ILINet and case count data. Negation terms to remove visits for GI illness incorrectly referred to as "flu-like" would be one useful change. The nature of visits for influenza at outpatient clinic versus hospitals is different, and it is possible this may account for the difference in the strength of correlations between the two data sources. Use of a different ILI syndrome definition for outpatient SyS data should be investigated.

Table 1. Pearson correlation coefficient values for influenza-like illness in three syndromic surveillance categories compared with ND ILINET and influenza case counts.

\begin{tabular}{|c|c|c|c|c|}
\hline \multicolumn{2}{|c|}{} & Outpatient III & Hospital ILI & Combined Outpatient and Hospital ILI \\
\hline $\begin{array}{c}\text { 2014-15 Season } \\
(\mathrm{n}=34 \text { weeks })\end{array}$ & ND ILINet & $0.44(\mathrm{p}=0.0090)$ & $0.77(\mathrm{p}<0.0001)$ & $0.76(\mathrm{p}<0.0001)$ \\
\cline { 2 - 6 } $\begin{array}{c}\text { 2015-16 Season } \\
(\mathrm{n}=33 \text { weeks })\end{array}$ & Neported cases & $0.44(\mathrm{p}=0.0098)$ & $0.92(\mathrm{p}<0.0001)$ & $0.90(\mathrm{p}<0.0001)$ \\
\cline { 2 - 6 } & Reported cases & $0.38(\mathrm{p}=0.0300)$ & $0.56(\mathrm{p}=0.0006)$ & $0.62(\mathrm{p}=0.0001)$ \\
\hline
\end{tabular}

\section{Keywords}

Syndromic Surveillance; Outpatient Data; Influenza-like Illness

\section{References}

1. Miller B, Kassenborg H, Dunsmuir W, et al. Syndromic Surveillance for Influenza-like Illness in Ambulatory Care Setting. Emerg Infect Dis. 2004 Oct;10(10):1806-1811.

2. Gren LH, Porucznik CA, Joy EA, et al. Point-of-Care Testing as an Influenza Surveillance Tool: Methodology and Lessons Learned from Implementation. Influenza Res Treat. 2013;2013:242970.

\section{*Jill K. Baber}

E-mail: jbaber@nd.gov 